\title{
CASES OF INTEREST \\ LABOUR COMPLICATED BY AN OVARIAN TUMOUR
}

\author{
Two Cases
}

SUMMARY: The management of labour in two patients with an ovarian mass is described.

\section{Introduction}

In patients from the hill communities in the region of Dharan, Nepal, disease pathology is frequently exaggerated through non-availability of early treatment. Patients are seen late in the course of their disease with gross pathology. This applies to ovarian cysts, both in the pregnant and non-pregnant.

Case 1

\section{Case reports}

Aged 24 years. This patient had had three normal deliveries at home. She booked at 32 weeks and was not seen again until 39 weeks when she presented in labour.

Findings. Patient was in strong labour. The lie was oblique with the breech presenting in the left iliac fossa. The cervix was displaced behind the pubic symphysis, pointing forwards and to the left. Cervix was $3.5 \mathrm{~cm}$ dilated. the membranes were intact.

Large fixed mass ballooning the Pouch of Douglas, extended upwards into the right pelvis. External version was not possible because the pelvic tumour had fixed the presenting part in the left iliac fossa.

The diagnosis was that of oblique lie in labour, caused by a large right sided ovarian tumour. Laparotomy was carried out.

Treatment. Lower segment caesarian section was performed. There was a large fixed mass replacing the right adnexum extending to the Pouch of Douglas. The sigmoid colon and rectum were densely adherent to the mass. The left adnexum was normal. An attempt was made to free the bowel but the adhesions were too dense to permit dissection without causing extensive damage. Instead, the tumour was incised and foul smelling pus drained. A suction drain was inserted into the large abscess cavity. Subsequent culture of the pus grew a pure strain of proteus.

The patient made an uneventful recovery.

\section{Case 2}

Aged 32 years. This patient presented about full term, with acute abdominal pain and breathlessness. The abdomen was grossly distended, tense and tender. A normal twin pregnancy was shown on X-ray. To relieve the acute pressure symptoms amniotomy was performed with the aim of reducing massive hydramnios. A small flow of liquor refuted the diagnosis of hydramnios. The abdomen was then tapped, and 28 pints of yellowish thick viscid fluid drained over 12 hours. Labour was then stimulated with Syntocinon. The patient delivered mature healthy twins and would not remain in hospital for further investigation and treatment.

At review 8 weeks later the gross abdominal distention had recurred so that laparotomy was necessary. 
Findings. A huge left sided ovarian cyst filled the abdominal cavity; the cyst wall was adherent to all the parietal peritoneum.

Treatment. The cyst was decompressed; 24 pints of thick jellowish viscid fluid were aspirated. Cyst wall was freed from peritoneum and the cyst pedicle cut and ligated.

Pathology. The pathological finding was pseudo mucinous cystadenoma.

\section{Comment}

The management of ovarian tumour complicating pregnancy and labour is well documented. Treatment is determined by the size of the tumour and the duration of the pregnancy. In the cases presented there was no choice of treatment. In one, caesarian section was required with exploration of an ovarian mass. In the other, decompression was urgently required for massive abdominal distension caused by an ovarian cyst.

\section{LIËUTENANT-COLONEL G. M. STEPHEN}

\section{TWO UNUSUAL CASES OF RETAINED PLACENTA}

\section{Introduction}

Both cases were treated by the author during one week while in the British Military Hospital, Dharan, Nepal. The stimulus to report the cases was provided by an article entitled "A Lost Placenta" (Beard and Johnson 1972). The patients were non-booked and were carried down to hospital from outlying villages.

\section{Case 1}

\section{Case reports}

A primigravida aged 22 years had delivered a macerated still birth at home the day before admission. The placenta was retained. She was in good general condition, apyrexial and did not show the features of gas gangrene. The uterine fundus was palpable above the umbilicus; the cervix was $2.5 \mathrm{~cm}$ dilated. Swabs were taken for aerobic and anaerobic culture.

Manual removal of the placenta under general anaesthetic was impossible, because it was only with difficulty that the whole hand could be introduced into the vagina by reason of 'hand-pelvis' disproportion. The procedure was abandoned.

The patient was discharged the following day on Chloramphenicol with the placenta in situ. She was seen again after a period of 6 months. She had had two normal periods since discharge, and had passed no large clots suggestive of placental tissues. The uterus was only slightly bulky, axial and mobile; the adnexae were normal. She had been working in the fields since departure from hospital and had no complaints.

\section{Case 2}

Aged 35 years, she was admitted with a retained placenta. She had delivered a macerated still birth 10 days before admission. Two untrained attempts had been made to deliver the placenta prior to admission. The patient was extremely toxic; temperature $103^{\circ} \mathrm{F}$, pulse $130 / \mathrm{min}$. The uterine fundus was palpable above the umbilicus; the whole uterus was hard and tender on palpation; the cervix was high and admitted one finger. 
There was a foul smelling discharge with the odour typical of gas gangrene. Swabs were taken and the patient prepared for laparotomy with the tentative diagnosis of puerperal gas gangrene.

Findings. On incising the grossly thickened peritoneum $300 \mathrm{ml}$ of foul smelling pus draine $d$. The uterus was covered with a thick fibrinous exudate and was adherent to the parietal peritoneum. There was a generalized purulent peritonitis.

Procedure. Sub-total hysterectomy was performed with the placenta in situ. An external drain was introduced into the cervical stump and the Pouch of Douglas.

The patient made an uneventful recovery. Clostridial culture was negative.

\section{Comment}

In Case 1, a primigravida, the untraditional expectant treatment of retained placenta left the patient with an unscarred uterus and child bearing potential. The alternative treatment would have been by hysterotomy leaving behind a dangerous scar for the future.

Radical treatment was required in Case 2 for a condition akin to a large perforated appendix with generalized purulent peritonitis.

\section{LIEUTENANT-COLONEL G. M. STEPHEN}

REFERENCE,

Beard, R. J. and Johnson, D. A. (1972). A Lost Placenta. J. Obstet Gynaec. Brit. Cwlth 79, 860. 